International Journal of Robotics and Automation (IJRA)

Vol. 9, No. 3, September 2020, pp. 153 159

ISSN: 2089-4856, DOI: 10.11591/ijra.v9i3.pp153-159

\title{
Forward kinematic analysis of Dobot using closed-loop method
}

\author{
Javier Sanjuan, Mohammad Rahman, Ivan Rulik
}

Mechanical Engineering Department, University of Wisconsin-Milwaukee, United States

\begin{tabular}{l} 
Article Info \\
\hline Article history: \\
Received Oct 23, 2019 \\
Revised Jan 12, 2020 \\
Accepted Mar 4, 2020
\end{tabular}

Keywords:

Dobot

Dynamical analysis

Forward kinematics

Parallel robots

Serial robots

\section{Corresponding Author:}

Javier Sanjuan,

Biorobotics Lab, USR 281,

Univesirty Services and Research Building, UW-Milwaukee,

115 East Reindl Way, Glendale, WI, 53212, United States.

Email: jsanjuan@uwm.edu

\begin{abstract}
Dobot is a hybrid robot that combines features from parallel and serial robots. Because of this characteristic, the robot excels for is reliability, allowing its implementation in diverse applications. Therefore, researchers have studied its kinematics to improve its capabilities. However, to the extent of our knowledge, no analysis has been reported taking into consideration the closed-loop configuration of Dobot. Thus, this article presents the complete analytical solution for the forward kinematics of Dobot, considering each link. The results are expected to be utilized in the development of a dynamical model that contemplates the dynamics of each element of the robot.
\end{abstract}

This is an open access article under the CC BY-SA license.

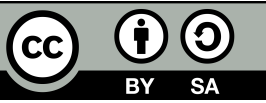

\section{INTRODUCTION}

Dobot is a 3 Degree of Freedom (DOF) type of commercial serial manipulator that posses numerous attractive features because of its hybrid serial and parallel configuration. One of its principal characteristics is the precision due to Dobot uses a four-bar linkage to actuate the rotation of each link. Additionally, Dobot utilizes a parallelogram mechanism to ensure that the orientation of the end-effector remains constant. Therefore, because of these features and its low cost, various researches have implemented Dobot in diverse applications such: 3D printing [1], electrochemical writing [2, 3], Pick and place [4-8], education [9], surgery [10], and even electric vehicle charging [11].

Because of the diverse applications of Dobot, researches have conducted different types of analysis on this robot. T. Cheng et al. analyzed the forward kinematics, inverse kinematics, and jacobian matrix of Dobot [11]. Moreover, O. Hock, et al. included an analysis of the pseudoinverse method to compute the inverse kinematics of Dobot numerically [12]. G. Yu et al. modified the end-effector of Dobot to included a gripper and a camera, to implement hand-eye calibration [13]. However, to the extent of our knowledge, no research has conducted the forward kinematics analysis of the linkage that composes the robot.

The forward kinematics analysis permits to obtain the kinematic of each link, which is a requirement to develop a detail dynamical model of Dobot. This type of model allows the implementation of robust control strategies such as inverse dynamics control $[14,15]$, permitting Dobot to be used in the application of high velocity while maintaining its precision [16]. Various researcher has worked in the computation of the direct kinematics for different parallel robots. Yujiong L., et al. presented the computation of the forward kinematics of an $\mathrm{H} 4$ parallel robot using a geometric approach [17]. Olaru D. obtained the forward and inverse 
kinematics of a 5DOF robot, between the relevant results from this research is the increasing of the robot precision due to the implementation of the computation of the forward kinematics in its control [18]. Jin S. K., et al. implemented the kinematic analysis of a 4DOF parallel robots for MRI-Guided percutaneous interventions [19]. Tang et al. study the kinematics of a novel 2R1T parallel mechanism [20]. Therefore, because of the advantages of a complete kinematical model, this research focuses on the computation of the forward kinematics of Dobot, considering the closed-loop configuration of each actuated joint. The results of the algorithm were validated using a CAD model, verifying the accuracy of the results.

The organization of the article is the following: Section 2 describes the principal joints of Dobot. Section 3 presents the kinematical diagram of Dobot, and explain the relation between links. Section 4 presents the computation of the forward kinematic of Dobot. Section 5 compares the results of the obtained equations with a CAD model of Dobot, to verify the accuracy of the results.

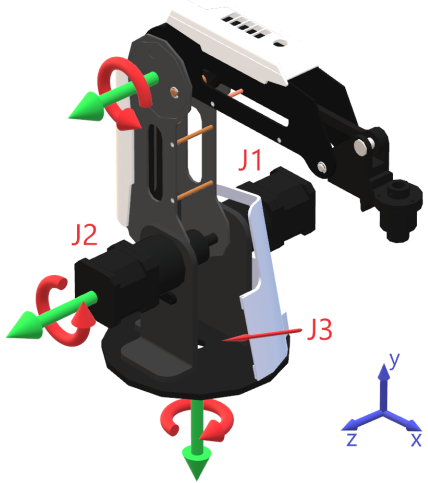

Figure 1. 3D model representation of DOBOT

\section{DOBOT DESCRIPTION}

The cad model of Dobot is presented in Figure 1. As can be seen, joints 1 and $2\left(q_{1} \& q_{2}\right)$ actuates in the same direction, therefore, the actuation of those joints, and their effect on the kinematics of the robot can be analyzed with a plane model. On the other hand, joint 3 rotates the robot in the $y$ direction, which changes the orientation Dobot. Since this rotation is perpendicular to the other actuated joints, this actuation is not considered in the analysis. Also, T. Cheng et al. presented a model to study the influence of this joint in the orientation of Dobot [11]. In Figure 2 the sectional view shows the mechanism inside DOBOT's case, where the variables explained in Section 2 can be related using Figures 2 and 3.

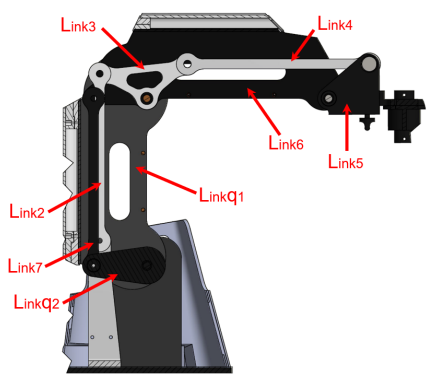

Figure 2. Sectional view of DOBOT

\section{KINEMATICS MODEL}

To facilitate the analysis of Dobot the kinematic diagram that presents all the links is obtained, as shown in Figure 3. Note that the actuated angles are represented by $q_{1}$ and $q_{2}$. Additionally, since dobot posses a parallel configuration, the actuation of the second joint is obtained by a four bar mechanism composed for links: $L_{q 2}, L_{7}$, and $L_{6}$, that transmit the movement from the base to this joint. The end effector is represent by 
point $P$, the orientation of this link is not directly actuated, and is dictated by a Watt's mechanism [21] that is composed for links: $L_{2}, L_{3}, L_{4}$ and $L_{5}$. Then, to verify that the kinematic representation of Dobot is correct, the Gruebler equation [22] is used to compute the DOF of the system, as follows:

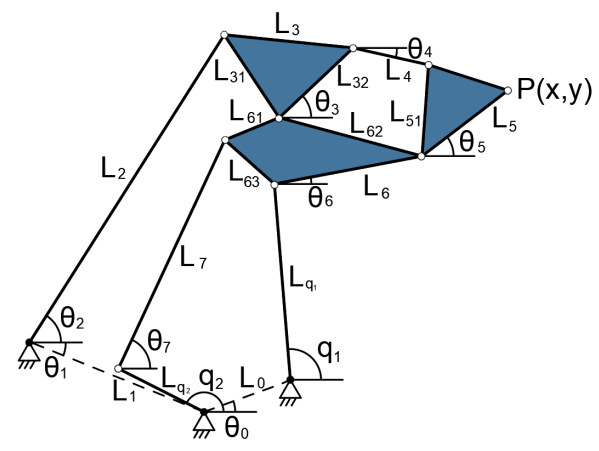

Figure 3. Kinematics model

$$
N=3 * L-2 * J
$$

Where $N$ is the number of DOF; $L$ is the number of links, and $J$ is the number of joints. Therefore, the number of DOF is 2 , which is the expected result.

\section{FORWARD KINEMATICS}

To obtain the forward kinematics it is used the closed-loop method [23]. This method requires the analysis of closed-loop vector to obtain the position equations. The number of closed-equations is determined by the use of the relation for Li et al. in [17], as follows:

$$
\begin{aligned}
d & =J-L \\
& =3
\end{aligned}
$$

Therefore, it is required to use three closed-loop equations to obtain the position of Dobot. The closed-loop used are presented in Figure 4.

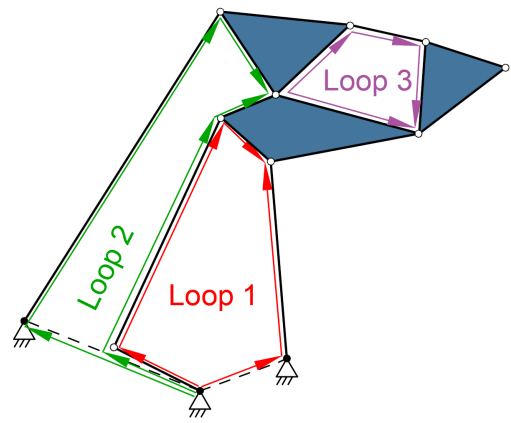

Figure 4. Closed-loops diagrams

For the first loop, the obtained equation is the following:

$$
\vec{L}_{q 2}+\vec{L}_{7}+\vec{L}_{63}=\vec{L}_{0}+\vec{L}_{q_{1}}
$$

Equation (3) is regrouped in terms of $L_{6}$ and expressed in vector form, as follows:

$$
L_{63}\left[\begin{array}{c}
\cos \theta_{6}+\beta_{3} \\
\sin \theta_{6}+\beta_{3}
\end{array}\right]=L_{0}\left[\begin{array}{c}
\cos \theta_{0} \\
\sin \theta_{0}
\end{array}\right]+L_{q 1}\left[\begin{array}{c}
\cos q_{1} \\
\sin q_{1}
\end{array}\right]-L_{q 2}\left[\begin{array}{c}
\cos q_{2} \\
\sin q_{2}
\end{array}\right]-L_{7}\left[\begin{array}{c}
\cos \theta_{7} \\
\sin \theta_{7}
\end{array}\right]
$$


Then, adding the square of the $x$ side and the square of the $y$ side, the term corresponding to $\theta_{6}$ is simplified. Thus, obtaining an expression that depends on $\theta_{7}, \theta_{q} 1, \theta_{q} 2$, and $\theta_{0}$. However, since $q_{1}$ and $q_{2}$ are entries of the system, and $\theta_{0}$ is a fix angle, the only variable of interest is $\theta_{7}$. Therefore, the resultant expression is expressed in term of $\theta_{7}$ as follows:

$$
A_{1} \cos \theta_{7}+A_{2} \sin \theta_{7}+A_{3}=0
$$

Where the terms $A_{i}$ are parameters that depends on the dimensions of Dobot, and known angles. Then, using the half tangent substitution $\left(\tan \frac{\theta_{7}}{2}=c_{1}\right)$, with $\cos \theta_{7}=\frac{1-c_{1}^{2}}{1+c_{1}^{2}}$ and $\sin \theta_{7}=\frac{2 c_{1}}{1+c_{1}^{2}},(5)$ is reduced to an algebraic expression, as follows:

$$
k_{1} C_{2}^{2}+k_{2} C_{2}+k_{3}=0
$$

Then, using the quadractic equation in (6), the following equation is obtained:

$$
C_{1}=\frac{-k_{2} \pm \sqrt{k_{2}^{2}-4 k_{1} k_{3}}}{2 k_{1}}
$$

Replacing $c_{1}$ for the tan $\frac{\theta_{7}}{2}$, the expression for $\theta_{7}$ is obtained:

$$
\theta_{7}=\operatorname{atan}\left(\frac{\sqrt{2} \sqrt{-\mathrm{L}_{q 1}{ }^{2} \mathrm{~L}_{q 2}{ }^{2}\left(\cos \left(2 q_{1}-2 q_{2}\right)-1\right)}+2 \mathrm{~L}_{q 1}{ }^{2} \sin \left(q_{1}\right)-2 \mathrm{~L}_{q 1} \mathrm{~L}_{q 2} \sin \left(q_{2}\right)}{2 \mathrm{~L}_{q 1}\left(\mathrm{~L}_{q 1}+\mathrm{L}_{q 1} \cos (q 1)-\mathrm{L}_{q 2} \cos \left(q_{2}\right)-\mathrm{L}_{q 2} \cos \left(q_{1}-q_{2}\right)\right)}\right)
$$

Now that the expression for $\theta_{7}$ is obtained, $\theta_{6}$ is computed from applying the dot product of 4 with unitary vector $\hat{i}=[1,0]^{T}$, and solving for $\theta_{6}$, generating:

$$
\theta_{6}=\pi-\operatorname{acos}\left(\frac{\mathrm{L}_{q 2} \cos \left(q_{2}\right)-\mathrm{L}_{q 1} \cos \left(q_{1}\right)+\mathrm{L}_{q 1} \cos \left(\theta_{7}\right)}{\mathrm{L}_{q 2}}\right)
$$

Analogously, the second loop equation is presented in the following:

$$
\vec{L}_{1}+\vec{L}_{2}+\vec{L}_{31}=\vec{L}_{q_{2}}+\vec{L}_{7}+\vec{L}_{61}
$$

The two variables to solve from (10) are $\theta_{2}$ and $\theta_{3}$, whose solutions are obtained applying the same methods for $\theta_{7}$ and $\theta_{6}$, respectively. Therefore, the solution for $\theta_{3}$ is presented below:

$$
\theta_{3}=2 \operatorname{atan}\left(\frac{-k_{12} \pm \sqrt{k_{12}^{2}-4 k_{11} k_{13}}}{2 k_{11}}\right)
$$

The values of $k_{11}, k_{12}$, and $k_{13}$ are presented in the Appendix section. The solution for $\theta_{2}$ is presented next.

$$
\theta_{2}=\operatorname{acos}\left(\frac{\mathrm{L}_{q 2} \cos \left(\beta_{1}+\theta_{6}\right)-\mathrm{L}_{q 2} \cos \left(\phi_{1}+\theta_{3}\right)+\mathrm{L}_{q 2} \cos \left(q_{2}\right)-\mathrm{L}_{q 2} \cos \left(\theta_{1}\right)+\mathrm{L}_{q 1} \cos \left(\theta_{7}\right)}{\mathrm{L}_{q 1}}\right)
$$

Then, using the same procedure as before, the third loop equation is the following:

$$
\vec{L}_{3}+\vec{L}_{4}=\vec{L}_{62}+\vec{L}_{51}
$$

From solving (13), the solution for $\theta_{5}$ is the following:

$$
\theta_{5}=-2 \operatorname{atan}\left(\frac{\sqrt{2} \sqrt{-L_{4}^{2} \mathrm{~L}_{q 2}^{2}\left(\cos \left(2 \theta_{3}-2 \theta_{6}\right)-1\right)}-2 \mathrm{~L}_{q 2}{ }^{2} \sin \left(\theta_{3}\right)+2 L_{4} \mathrm{~L}_{q 2} \sin \left(\theta_{6}\right)}{2 \mathrm{~L}_{q 2}\left(\mathrm{~L}_{q 2}-L_{4} \cos \left(\theta_{6}\right)+\mathrm{L}_{q 2} \cos \left(\theta_{3}\right)-L_{4} \cos \left(\theta_{3}-\theta_{6}\right)\right)}\right)
$$

And for $\theta_{4}$ :

$$
\theta_{4}=\left(\operatorname{acos}\left(\frac{\mathrm{L}_{q 2} \cos \left(\gamma_{1}+\theta_{5}\right)+L_{4} \cos \left(\theta_{6}\right)-\mathrm{L}_{q 2} \cos \left(\theta_{3}\right)}{L_{4}}\right)\right)
$$


Lastly, using the fourth loop as presented in Figure 5, the values of $x$ and $y$ are obtained using the following expression:

$$
\begin{gathered}
\mathrm{L}_{q 1} \cos \left(q_{1}\right)+L_{4} \cos \left(\theta_{6}\right)+L_{5} \cos \left(\theta_{5}\right)=x \\
\mathrm{~L}_{q 1} \sin \left(q_{1}\right)+L_{4} \sin \left(\theta_{6}\right)+L_{5} \sin \left(\theta_{5}\right)=y
\end{gathered}
$$

Note that (8), (9), (11), (12), (14), (15), and (16) were simplified considering the dimensional parameters presented in Table 1.

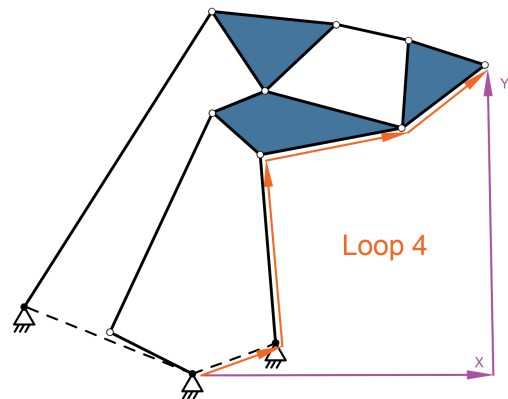

Figure 5. Fourth loop

Table 1. Dimensional parameters on DOBOT

\begin{tabular}{cc}
\hline Parameter & Values \\
\hline$L_{63}, L_{q 2}, L_{1}, L_{31}, L_{61}, L_{3}, L_{51}$ & $43[\mathrm{~mm}]$ \\
$L_{7}, L_{q 1}, L_{2}$ & $135[\mathrm{~mm}]$ \\
$L_{4}, L_{62}$ & $147[\mathrm{~mm}]$ \\
$L_{0}$ & $0[\mathrm{~mm}]$ \\
$\theta_{0}, \beta_{1}, \beta_{2}, \beta_{3}$ & $0[\mathrm{rad}]$ \\
$\theta_{1}$ & $2.66[\mathrm{rad}]$ \\
$\phi_{1}$ & $5.1[\mathrm{rad}]$ \\
$\gamma_{1}$ & $0.7[\mathrm{rad}]$ \\
\hline
\end{tabular}

\section{RESULTS}

To validate the kinematics model and evaluate its accuracy, a comparison was done using the CAD obtained from $D O B O T^{\odot}{ }^{\circ}$. Both, the results from the CAD model and the Kinematic model are presented in Table. When comparing the results it is clear that the computed values and the CAD model present the same results. Therefore, the forward kinematic analysis is accurate.

Table 2. Test of the Kinematic Model

\begin{tabular}{ccc}
\hline Variables & ${\text { CAD measure }\left[{ }^{\circ} \text { ] }\right.}$ & Kinematic Model $\left.{ }^{\circ}\right]^{-}$ \\
\hline$q_{1}$ & 44 & 44 \\
$q_{2}$ & 150 & 150 \\
$\theta_{2}$ & 44 & 44 \\
$\theta_{3}$ & 40.28 & 40.2823 \\
$\theta_{4}$ & 30 & 30 \\
$\theta_{5}$ & 0.28 & 0.2823 \\
$\theta_{6}$ & 30 & 30 \\
$\theta_{7}$ & 44 & 44 \\
\hline
\end{tabular}

\section{CONCLUSIONS}

This paper presents the forward kinematic analysis of Dobot, a 3DOF hybrid robot that is posses both, a serial and parallel configuration. The equations were expressed using the closed-loop method and were solved analytically by the application of the tangent half-angle substitution. This result is expected to be utilized in the computation of the dynamics of Dobot, to implement elaborated control strategies such as inverse dynamic control. The accuracy of the equations was also validated using a CAD model, obtaining an easy to utilize a set of equations to obtain the kinematic of Dobot as a function of the joint angles. 


\section{APPENDIX}

The following equations present the values of $k_{11}, k_{12}$, and $k_{13}$.

$$
\begin{aligned}
& k_{11}=2 \mathrm{~L}_{q 2}^{2} \cos \left(\phi_{1}-q_{2}\right)-2 \mathrm{~L}_{q 2}^{2} \cos \left(\phi_{1}-\theta_{1}\right)+2 \mathrm{~L}_{q 2}^{2} \cos \left(\phi_{1}-\theta_{6}\right)-2 \mathrm{~L}_{q 2}{ }^{2} \cos \left(q_{2}-\theta_{1}\right) \\
& +2 \mathrm{~L}_{q 2}{ }^{2} \cos \left(q_{2}-\theta_{6}\right)-2 \mathrm{~L}_{q 2}{ }^{2} \cos \left(\theta_{1}-\theta_{6}\right)+4 \mathrm{~L}_{q 2}{ }^{2}-2 \mathrm{~L}_{q 1} \mathrm{~L}_{q 2} \cos \left(\theta_{1}-\theta_{7}\right) \\
& +2 \mathrm{~L}_{q 1} \mathrm{~L}_{q 2} \cos \left(\theta_{6}-\theta_{7}\right)+2 \mathrm{~L}_{q 1} \mathrm{~L}_{q 2} \cos \left(\phi_{1}-\theta_{7}\right)+2 \mathrm{~L}_{q 1} \mathrm{~L}_{q 2} \cos \left(q_{2}-\theta_{7}\right) \\
& k_{12}=4 \mathrm{~L}_{q 2}{ }^{2} \sin \left(\phi_{1}-q_{2}\right)-4 \mathrm{~L}_{q 2}^{2} \sin \left(\phi_{1}-\theta_{1}\right)+4 \mathrm{~L}_{q 2}^{2} \sin \left(\phi_{1}-\theta_{6}\right)+4 \mathrm{~L}_{q 1} \mathrm{~L}_{q 2} \sin \left(\phi_{1}-\theta_{7}\right) \\
& k_{13}=2 \mathrm{~L}_{q 2}{ }^{2} \cos \left(\phi_{1}-\theta_{1}\right)-2 \mathrm{~L}_{q 2}{ }^{2} \cos \left(\phi_{1}-q_{2}\right)-2 \mathrm{Lq} 2^{2} \cos \left(\phi_{1}-\theta_{6}\right)-2 \mathrm{~L}_{q 2}{ }^{2} \cos \left(q_{2}-t_{1}\right) \\
& +2 \mathrm{~L} q 2^{2} \cos \left(q_{2}-t_{6}\right)-2 \mathrm{~L} q 2^{2} \cos \left(t_{1}-t_{6}\right)+4 \mathrm{~L} q 2^{2}-2 \mathrm{~L}_{q 1} \mathrm{~L}_{q 2} \cos \left(\theta_{1}-\theta_{7}\right)
\end{aligned}
$$

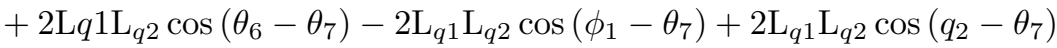

\section{REFERENCES}

[1] L. Wu, J. Yang, X. Zhang, and Y. Chen, "Multi manipulator cooperative 3d printing based on dobot manipulator," in IOP Conference Series: Materials Science and Engineering, vol. 382, no. 4. IOP Publishing, pp. 042040, 2018.

[2] X. Zhai, X. Zou, J. Shi, X. Huang, Z. Sun, Z. Li, Y. Sun, Y. Li, X. Wang, M. Holmes et al., "Amineresponsive bilayer films with improved illumination stability and electrochemical writing property for visual monitoring of meat spoilage," Sensors and Actuators B: Chemical, p. 127130, 2019.

[3] R. Guo, X. Sun, B. Yuan, H. Wang, and J. Liu, "Magnetic liquid metal (fe-egain) based multifunctional electronics for remote self-healing materials, degradable electronics, and thermal transfer printing," Advanced Science, 2019.

[4] I. Md Rasedul, R. Md Arifur, M. A. uz Zaman, and H. R. Mohammad, "Cartesian trajectory based control of dobot robot," in Proceedings of the International Conference on Industrial Engineering and Operations Management. IEOM Society International, pp. 1507-1517, 2019.

[5] F. Nagata, Y. Seda, K. Hamada, S. Suzuki, A. Otsuka, T. Ikeda, H. Ochi, K. Watanabe, M. K. Habib, and T. Kusano, "Outline font handler for industrial robots," in 2018 IEEE International Conference on Mechatronics and Automation (ICMA). IEEE, pp. 1823-1828, 2018.

[6] P. Jaskólski and K. Nadolny, "Characteristic of process flow in modular didactic production system for gear trains," Journal of Mechanical and Energy Engineering, vol. 3, no. 2, pp. 115-120, 2019.

[7] P. Urhal, A. Weightman, C. Diver, and P. Bartolo, "Robot assisted additive manufacturing: A review," Robotics and Computer-Integrated Manufacturing, vol. 59, pp. 335-345, 2019.

[8] R. Chen, R. Song, Z. Zhang, L. Bai, F. Liu, P. Jiang, D. Sindersberger, G. J. Monkman, and J. Guo, "Bio-inspired shape-adaptive soft robotic grippers augmented with electroadhesion functionality," Soft robotics, 2019.

[9] D. Scaradozzi, L. Screpanti, and L. Cesaretti, "Towards a definition of educational robotics: a classification of tools, experiences and assessments," in Smart Learning with Educational Robotics. Springer, pp. 63-92, 2019.

[10] T. Cheng, W. Li, C. S. H. Ng, P. W. Y. Chiu, and Z. Li, "Visual servo control of a novel magnetic actuated endoscope for uniportal video-assisted thoracic surgery," IEEE Robotics and Automation Letters, vol. 4, no. 3, pp. 3098-3105, 2019.

[11] M. Behl, J. DuBro, T. Flynt, I. Hameed, G. Lang, and F. Park, "Autonomous electric vehicle charging system," in 2019 Systems and Information Engineering Design Symposium (SIEDS). IEEE, pp. 1-6, 2019.

[12] O. Hock and J. Šedo, "Forward and inverse kinematics using pseudoinverse and transposition method for robotic arm dobot," in Kinematics. IntechOpen, 2017.

[13] G. Yu, Y. Liu, X. Han, and C. Zhang, "Objects grasping of robotic arm with compliant grasper based on vision," in Proceedings of the 2019 4th International Conference on Automation, Control and Robotics Engineering. ACM, pp. 62, 2019.

[14] P. K. Jamwal, S. Hussain, M. H. Ghayesh, and S. V. Rogozina, "Adaptive impedance control of parallel ankle rehabilitation robot," Journal of Dynamic Systems, Measurement, and Control, vol. 139, no. 11, pp. 111006, 2017. 
[15] M. Rachedi, M. Bouri, and B. Hemici, "Robust control of a parallel robot," in 2015 International Conference on Advanced Robotics (ICAR). IEEE, pp. 428-433, 2015.

[16] H. D. Taghirad, Parallel robots: mechanics and control. CRC press, 2013.

[17] Y. Liu, M. Kong, N. Wan, and P. Ben-Tzvi, "A geometric approach to obtain the closed-form forward kinematics of h4 parallel robot," Journal of Mechanisms and Robotics, vol. 10, no. 5, pp. 051013, 2018.

[18] O. D. Adrian, O. A. Serban, and M. C. Niculae, "Forward and inverse kinematics applied to the parallel robot's structure," International Journal of Modeling and Optimization, vol. 7, no. 6, 2017.

[19] J. S. Kim, D. Levi, R. Monfaredi, K. Cleary, and I. Iordachita, "A new 4-dof parallel robot for mri-guided percutaneous interventions: Kinematic analysis," in 2017 39th Annual International Conference of the IEEE Engineering in Medicine and Biology Society (EMBC). IEEE, pp. 4251-4255, 2017.

[20] H. Tang, D. Zhang, S. Guo, H. Qu, and G. Huang, "Kinematics analysis of a novel 2r1t parallel mechanism," International Journal of Robotics and Automation, vol. 33, no. 2, 2018.

[21] E. Primrose, F. Freudenstein, and B. Roth, "Six-bar motion i. the watt mechanism," Archive for Rational Mechanics and Analysis, vol. 24, no. 1, pp. 22-41, 1967.

[22] B. Heling, B. Schleich, and S. Wartzack, "Robust-design-optimization of mechanisms based on kinematic requirements considering uncertainties," Procedia CIRP, vol. 75, pp. 27-32, 2018.

[23] Q. Zhe, L. Xiao-Chu, and Z. Zhuan, "Delta parallel robot based on crank-slider mechanism," International Journal of Robotics and Automation (IJRA), vol. 6, no. 2, pp. 112-120, 2017.

\section{BIOGRAPHIES OF AUTHORS}
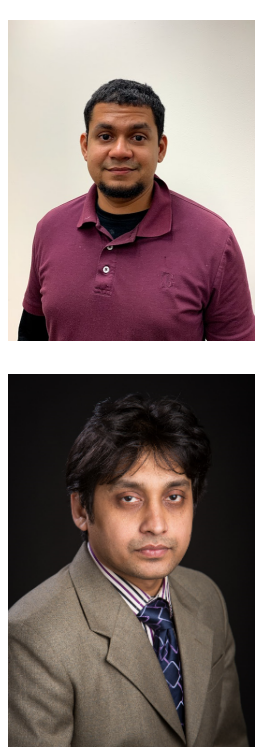

Javier Sanjuan is a Ph.D. student at the BioRobotics Lab, University of Wisconsin-Milwaukee. He obtained his masters and bachelors degrees in Mechanical Engineering at Universidad del Norte, Cololmbia (2012-2016). His research interest includes dynamics, control, and parallel robots. In Mechanisms and Machine Theory Journal, IEEE Latin America, and other scientific publications, he has served as invited reviewer. Besides, he is one of the founders of the Vertical Integrated Projects (VIP) student association in Universidad del Norte, Colombia.

Further info on his homepage: https://www.researchgate.net/profile/Javier ${ }_{S}$ anjuan 5

Mohammad Rahman is with the Mechanical and Biomedical Engineering Department, University of Wisconsin-Milwaukee, WI, USA. As Director of the BioRobotics Lab at the University of Wisconsin-Milwaukee, he brings the resources and expertise of an interdisciplinary R\&D team. For more than 15 years he has been researching bio-mechatronics/bio-robotics with emphasis on the design, development and control of wearable robots to rehabilitate and assist elderly and physically disabled individuals who have lost their upper-limb function or motion due to stroke, cardiovascular disease, trauma, sports injuries, occupational injuries, and spinal cord injuries. He received a BSc Engineering (mechanical) degree from Khulna University of Engineering \& Technology, Bangladesh in 2001, a Master of Engineering (bio-robotics) degree from Saga University, Japan in 2005 and a $\mathrm{PhD}$ in Engineering (bio-robotics) from École de technologie supérieure (ETS), Université du Québec, Canada in 2012. He worked as a postdoctoral research fellow in the School of Physical \& Occupational Therapy, McGill University (2012-2014). His research interests are in bio-robotics, exoskeleton robot, intelligent system and control, mobile robotics, nonlinear control, control using biological signal such as electromyogram signals.

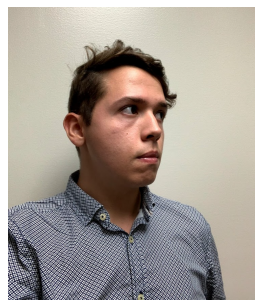

Ivan Rulik is a senior Electrical and Biomedical Engineering international student from Universidad de Colombia, and will get his degree in 2020. He is currently a Research Assistant in the Mechanical Engineering Department at University of Wisconsin-Milwaukee, working in the BioRobotics lab. He brings expertise in programming using Matlab and designing in CAD with Inventor, AutoCAD and Solidworks. His research interests include robotics, microrobotics, medicine, rehabilitation devices and electromagnetic manipulation at small scales $\mathrm{He}$ is affiliated with IEEE as student member. Besides, he is also involved in the IEEE Industry Applications Society Student Branch from Universidad de los Andes, Colombia. 